\title{
A systematic review and meta-analysis of treatment for hepatorenal syndrome with traditional Chinese medicine
}

\author{
Tingxue Song ${ }^{1,2}$, Xiaozhong Guo ${ }^{1 \#}$, Lichun Shao ${ }^{2}$, Mingyu Sun ${ }^{3}$, Fernando Gomes Romeiro ${ }^{4}$, Dan $\mathrm{Han}^{1}$, \\ Wenchun Bao ${ }^{1}$, Xingshun $\mathbf{Q i}^{1 \#}$
}

${ }^{1}$ Liver Cirrhosis Study Group, Department of Gastroenterology, General Hospital of Shenyang Military Area, Shenyang 110016, China; ${ }^{2}$ Department of Gastroenterology, No. 463 Hospital of Chinese PLA, Shenyang 110000, China; ${ }^{3}$ Institute of Liver Diseases, Shuguang Hospital Affiliated to Shanghai University of Traditional Chinese Medicine, Shanghai 201203, China; ${ }^{4}$ Botucatu Medical School, UNESP-Universidade Estadual Paulista, São Paulo State, Brazil

Contributions: (I) Conception and design: X Qi, X Guo; (II) Administrative support: None; (III) Provision of study materials or patients: T Song, X Qi, X Guo, L Shao; (IV) Collection and assembly of data: T Song, X Qi, X Guo; (V) Data analysis and interpretation: T Song, X Qi, X Guo, L Shao; (VI) Manuscript writing: All authors; (VII) Final approval of manuscript: All authors.

"These authors contributed equally to this work.

Correspondence to: Dr. Xingshun Qi; Prof. Xiaozhong Guo. Liver Cirrhosis Study Group, Department of Gastroenterology, General Hospital of Shenyang Military Area, Shenyang 110016, China. Email: xingshunqi@126.com; guo_xiao_zhong@126.com.

Background: Hepatorenal syndrome (HRS) is a life-threatening complication of end-stage liver diseases. It has been reported that traditional Chinese medicine (TCM) may improve liver function, delay disease progression, alleviate symptoms, and improve quality of life in HRS patients. The study aims to systematically review the efficacy of TCM for the treatment of HRS.

Methods: Publications were searched electronically from China National Knowledge Infrastructure (CNKI), Wanfang, VIP, PubMed, and EMBASE databases. Odds ratio (OR) and standardized mean difference (SMD) with 95\% confidence interval (CI) were calculated. Heterogeneity was assessed. The Cochrane Collaboration's tool was used to assess the risk of bias.

Results: Fourteen randomized controlled trials involving 788 patients with HRS were included. Random generation sequence was reported in only two studies. Blinding was not used in any study. Compared to conventional treatment without TCM, TCM led to a significant survival benefit during hospitalization (OR: 0.18; 95\% CI: 0.08-0.39; P<0.0001), a significantly higher complete response (OR: 3.20; 95\% CI: 2.06-4.97; $\mathrm{P}<0.00001$ ), and a significantly lower no response (OR: 0.20; 95\% CI: 0.14-0.30; $\mathrm{P}<0.00001$ ). Partial response was not significantly different between the two groups (OR: 1.39; 95\% CI: 0.90-2.15; P=0.14). Regardless of TCM, blood urea nitrogen and abdominal circumference were significantly decreased, and urine volume was significantly increased after treatment. Compared to conventional treatment without TCM, TCM led to a significantly lower serum creatinine, blood urea nitrogen, bilirubin, plasma ammonia, and abdominal circumference and significantly higher urine volume after treatment. There was significant heterogeneity.

Conclusions: TCM might have a better survival and a higher complete response in patients with HRS. However, the quality of published studies was unsatisfactory.

Keywords: Traditional Chinese medicine (TCM); hepatorenal syndrome (HRS); response; liver function; renal function; terlipressin

Received: 24 May 2018; Accepted: 09 August 2018; Published: 22 August 2018.

doi: $10.21037 / \operatorname{tgh} .2018 .08 .02$

View this article at: http://dx.doi.org/10.21037/tgh.2018.08.02 


\section{Introduction}

Hepatorenal syndrome (HRS) is a lethal complication of end-stage liver diseases, which is a functional kidney injury developing as a consequence of the severe reduction in the renal perfusion secondary to splanchnic arterial vasodilation (1). HRS can occur spontaneously or is secondary to hypovolemia and bacterial infection $(2,3)$. The prognosis of HRS remains dismal with a median survival time of approximately 3 months (4). Terlipressin, noradrenaline, midodrine, and octreotide have been used for the treatment of HRS (5-7), which can result in splanchnic vasoconstriction and then lead to an increase in effective circulating blood volume and renal blood flow $(8,9)$.

According to the traditional Chinese medicine (TCM) theory, HRS, which is called as bulging, is caused by the "qi" stagnation, blood stasis, and phlegm-retained fluid. The current TCM expert consensus suggests that TCM may improve liver function, delay disease progression, alleviate symptoms, and improve quality of life in HRS patients (10).

A systematic review and meta-analysis aimed to evaluate the efficacy of TCM for the treatment of HRS.

\section{Methods}

\section{Registration}

The number of registration in PROSPERO was CRD42017076055.

\section{Search strategy}

Relevant publications were searched electronically from the China National Knowledge Infrastructure (CNKI), Wanfang, VIP, PubMed, and EMBASE databases. The search items were "hepatorenal syndrome", "traditional Chinese medicine", "herb", and "random". The date of last search was September 9, 2017.

\section{Paper selection}

The eligibility criteria included: (I) patients with HRS; (II) TCM with and without conventional therapy as the TCM group; (III) conventional therapy without TCM as the control group; (IV) randomized controlled trials (RCTs); and $(\mathrm{V})$ studies reporting the efficacy of TCM.

Exclusion criteria were as follows: (I) duplicate publications; (II) reviews; (III) basic researches; (IV) systematic reviews and meta-analyses; (V) irrelevant topics;
(VI) unable to extract the data regarding patients with HRS; and (VII) catalogues, indexes, and conference reports. No language and publication status were limited.

\section{Data extraction}

Primary data were extracted, including characteristics of studies, baseline characteristics of patients, response of HRS, and changes of biomedical variables after the treatment. The characteristics of studies were as follows: first author, study design, year of publication, region, enrollment period, number of patients in TCM/control group, methods of intervention, treatment period, and follow-up time. The characteristics of patients were as follows: age, gender, serum creatinine, blood urea nitrogen, bilirubin, urine volume, and abdominal circumference.

\section{Risk of bias assessment}

The Cochrane Collaboration's tool to assess the risk of bias was employed. It includes 7 domains: random sequence generation, allocation concealment, blinding of participants and personnel, blinding of outcome assessment, incomplete outcome data, selective reporting, and other bias.

\section{Endpoints}

Outcomes of interest were: (I) the death of patients with HRS; (II) the response; and (III) the changes of biomedical variables. Response was divided into complete, partial, and no response according to the definitions established by original articles.

\section{Statistical analysis}

The meta-analyses were performed by the Review Manager 5.3 (Copenhagen: The Nordic Cochrane Centre, The Cochrane Collaboration, Copenhagen, Denmark) and Stata version 12 (StataCorp, College Station, Texas, USA). Continuous data were expressed as mean \pm standard deviation (SD). Random-effect model was employed. Odds ratio $(\mathrm{OR})$ with $95 \%$ confidence interval $(\mathrm{CI})$ was calculated for binary variables. Standardized mean difference (SMD) with $95 \%$ CI was calculated for continuous variables. $\mathrm{P}<0.05$ was considered to indicate statistical significance. Heterogeneity was quantified using the Cochrane Q-test and the $\mathrm{I}^{2}$ statistics. $\mathrm{P}<0.1$ or $\mathrm{I}^{2}>50 \%$ was considered to indicate a statistically significant heterogeneity. Subgroup, 


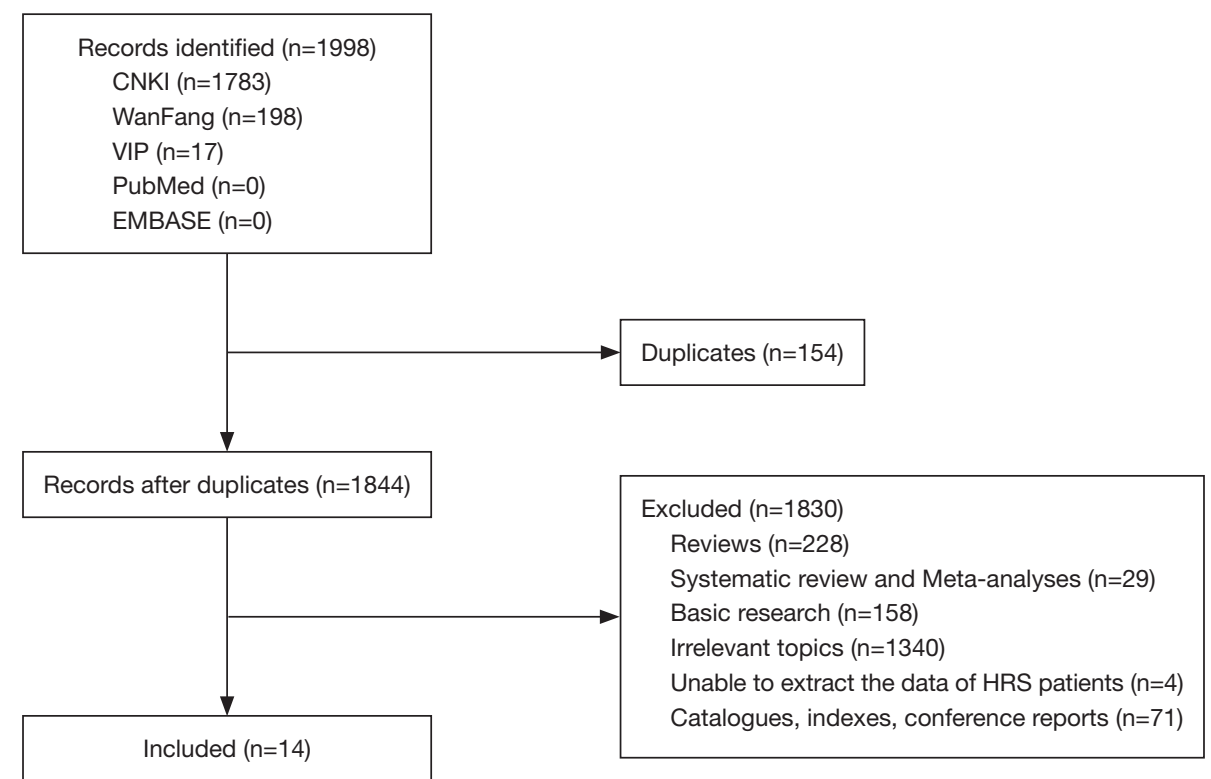

Figure 1 Flow chart of selection of publications.

sensitivity, and meta-regression analyses were used to analyze the source of heterogeneity. Subgroup analyses were performed according to the type of HRS and diagnostic criteria for HRS. As the number of included studies was $\geq 9$, we conducted meta-regression analyses. In meta-regression analyses, the covariates included publication year, type of HRS, and diagnostic criteria for HRS.

\section{Results}

\section{Characteristics of studies}

A total of 1998 studies were identified. Fourteen studies were included (11-24) (Figure 1). The sample size ranged from 25 to 140 . The publication year ranged from 2004 to 2017. The publication regions were all in China. Only two studies included patients with type 2 HRS alone, and others included patients with unclassified type of HRS. Characteristics of studies were summarized in Table 1. The diagnosis of HRS was based on the International Club of Ascites (ICA) in 7 studies or other diagnostic criteria in 5 studies and was unspecified in 2 studies.

\section{Characteristics of patients}

Age, gender, and urine volume were provided in 9 studies. Serum creatinine, blood urea nitrogen, and bilirubin were presented in 12, 11, and 5 studies, respectively. In 8 studies, the underlying liver disease was liver cirrhosis alone. In 3 studies, the underlying liver disease included liver cirrhosis, liver cancer, or severe hepatitis. In 3 other studies, the underlying liver disease remained unclear. Five studies provided information regarding etiology of liver disease. Viral hepatitis was the major etiology of liver disease followed by alcohol abuse. Characteristics of patients were summarized in Table S1.

\section{Risk of bias}

Only 2 studies reported the random sequence generation, of which one had a high risk and another had a low risk. All studies had low risks of attrition bias and reporting bias. Other risks of bias were unclear in most of studies (Figure S1).

\section{Outcomes}

\section{Death}

Six studies with 279 patients were included in the metaanalysis regarding in-hospital death (Figure 2A). TCM led to a significant survival benefit (OR: $0.18,95 \%$ CI: $0.08-$ $0.39, \mathrm{P}<0.0001)$. There was no significant heterogeneity $\left(\mathrm{P}=0.18, \mathrm{I}^{2}=32 \%\right)$.

\section{Response}

Ten studies with 685 patients were included in the meta- 


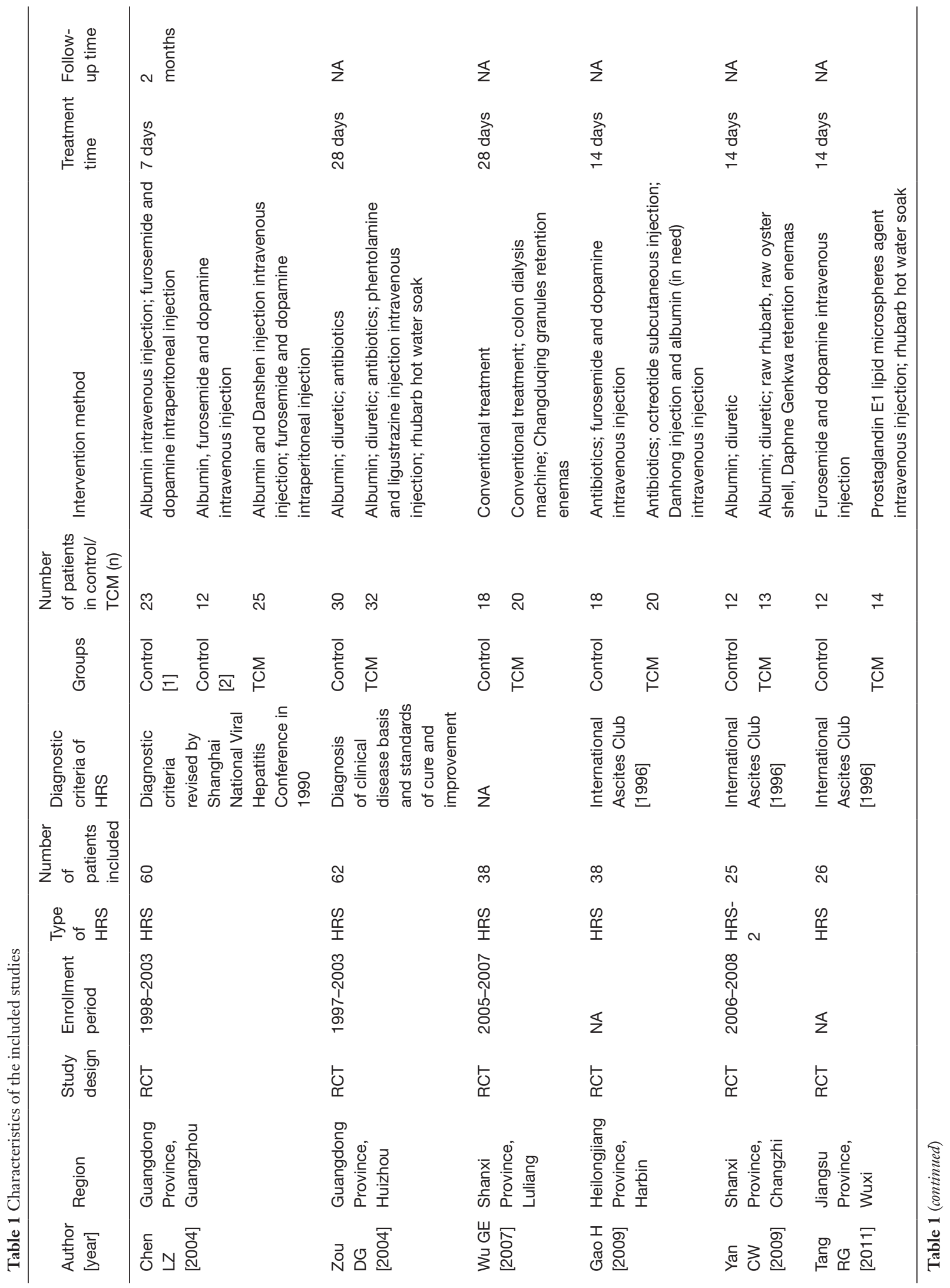




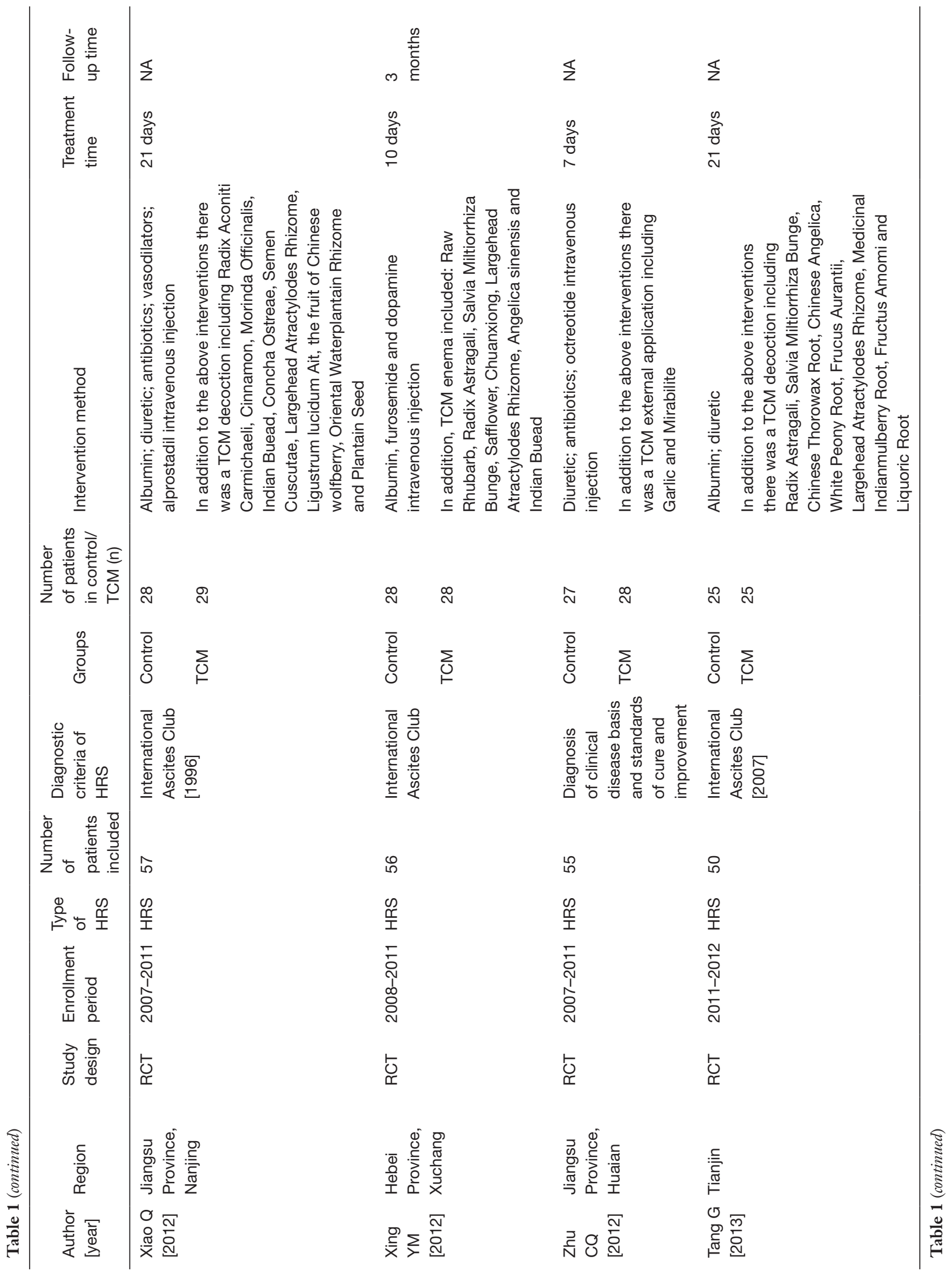




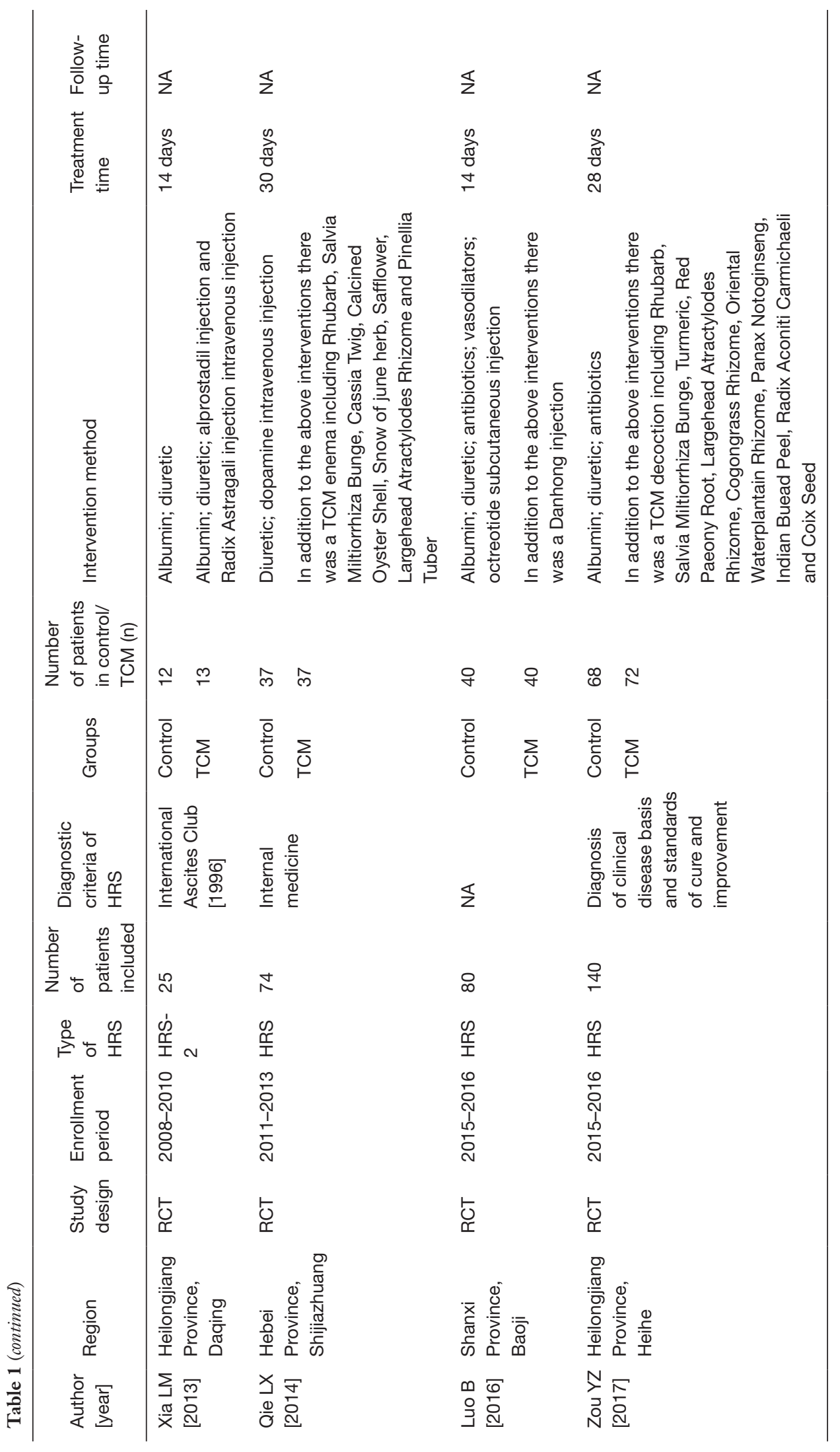


A

\begin{tabular}{|c|c|c|c|c|c|}
\hline \multirow[b]{2}{*}{ Study or Subgroup } & \multicolumn{2}{|c|}{ Experimental } & \multicolumn{2}{|c|}{ Control } & \multirow[b]{2}{*}{ Weight } \\
\hline & & Total & & Total & \\
\hline Chen LZ (1) (2004) & 5 & 25 & 12 & 23 & $21.2 \%$ \\
\hline Chen LZ (2) (2004) & 5 & 25 & 11 & 12 & $9.7 \%$ \\
\hline Tang RG (2011) & 2 & 14 & 12 & 12 & $5.6 \%$ \\
\hline Xia LM (2013) & 1 & 13 & 2 & 12 & $8.0 \%$ \\
\hline Xing YM (2012) & 5 & 28 & 12 & 28 & $22.2 \%$ \\
\hline Yan CW (2009) & 1 & 13 & 2 & 12 & $8.0 \%$ \\
\hline Zou DG (2004) & 11 & 32 & 21 & 30 & $25.4 \%$ \\
\hline Total $(95 \% \mathrm{Cl})$ & & 150 & & 129 & $100.0 \%$ \\
\hline Total events & 30 & & 72 & & \\
\hline \multicolumn{6}{|c|}{ Heterogeneity: $\mathrm{Tau}^{2}=0.34 ; \mathrm{Chi}^{2}=8.84, \mathrm{df}=6(\mathrm{P}=0.18) ; \mathrm{I}^{2}=32 \%$} \\
\hline Test for overall effect: & $=4.32($ & $<0.000$ & & & \\
\hline
\end{tabular}

B

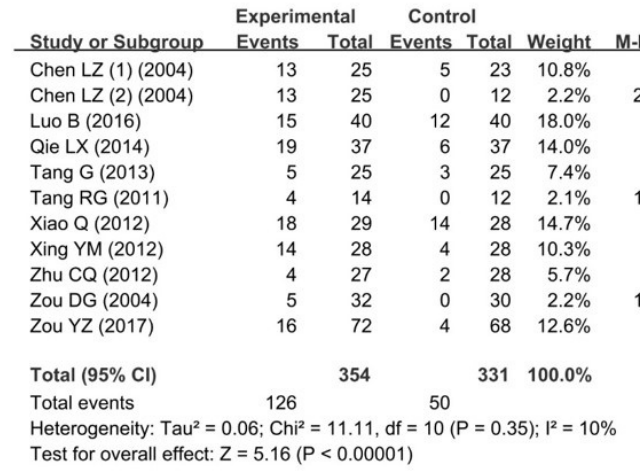

Odds Ratio $3.90[1.10,13.80]$ $27.00[1.44,505.40]$

$1.40[0.55,3.55]$ $5.45[1.84,16.16]$ $5.45[1.84,16.16]$
$1.83[0.39,8.67]$ $10.71[0.52,222.81]$ $1.64[0.57,4.70]$ $6.00[1.65,21.84]$ $2.26[0.38,13.51]$ $12.20[0.64,230.91]$ $4.57[1.44,14.48]$

$3.20[2.06,4.97]$

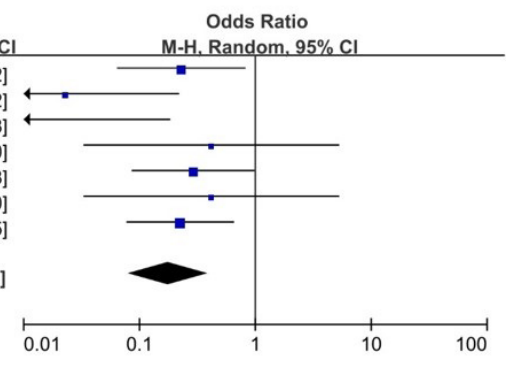

\section{C}

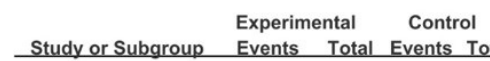

Chen LZ (1) (2004)

Chen LZ (2) (2004)

Luo B (2016)

Qie LX (2014)

Tang G (2013)

Tang RG (2011)

Xiao Q (2012)

Xing YM (2012)

Zhu CQ (2012)

Zou DG (2004)

Zou YZ (2017)

Total $(95 \% \mathrm{Cl})$

Total events

Heterogeneity: $\mathrm{Tau}^{2}=0.23 \cdot \mathrm{Chi}^{2}=17.85, \mathrm{df}=10(\mathrm{P}=0.06) ; \mathrm{I}^{2}=44 \%$

Test for overall effect: $Z=1.49(P=0.14)$

Odds Ratio

Odds Ratio M-H. Random. $95 \%$ Cl

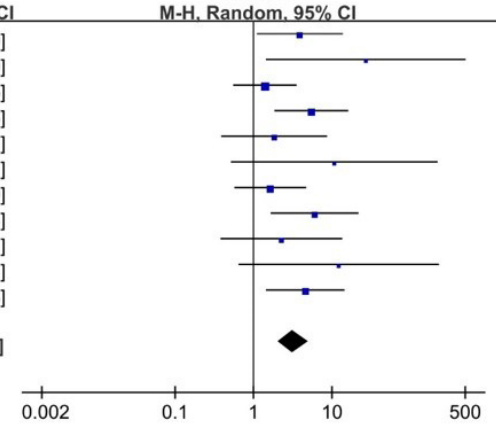

Random. $95 \% \mathrm{CI}$

$0.61[0.19,1.99]$

$0.66[0.16,2.73]$

$2.08[0.84,5.14]$

$0.57[0.22,1.45]$

$1.40[0.45,4.35]$

$1.87[0.39,8.89]$

$1.46[0.40,5.31]$

$0.56[0.19,1.62]$

$2.38[0.78,7.20]$

$3.34[1.16,9.61]$

$2.92[1.45,5.90]$

$1.39[0.90,2.15]$

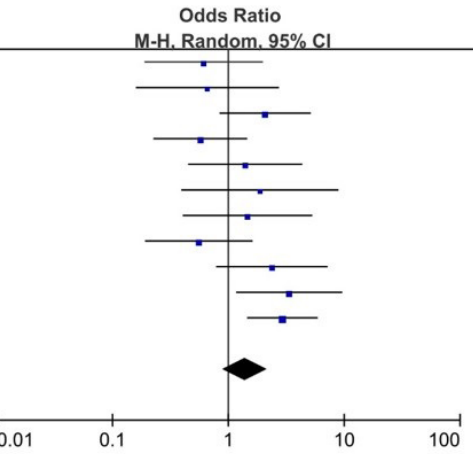

-H. Random, $95 \% \mathrm{Cl}$

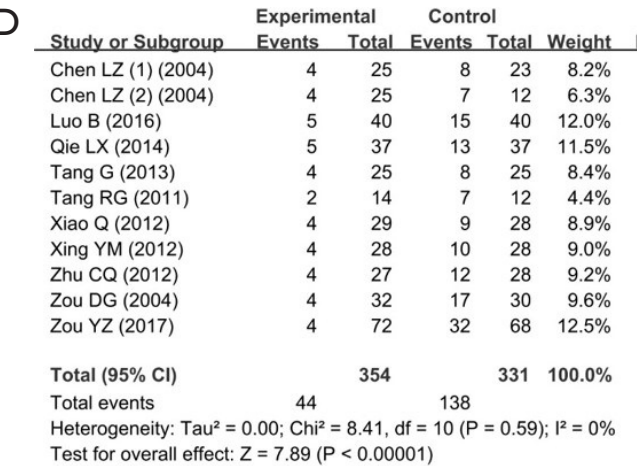

Odds Ratio $0.36[0.09,1.41]$ $0.14[0.03,0.65]$ $0.24[0.08,0.74]$ $0.40[0.10,1.58]$ $0.12[0.02,0.79]$ $0.34[0.09,1.26]$ $0.30[0.08,1.11]$ $0.23[0.06,0.85]$ $0.11[0.03,0.39]$ $0.07[0.02,0.20]$

$0.20[0.14,0.30]$

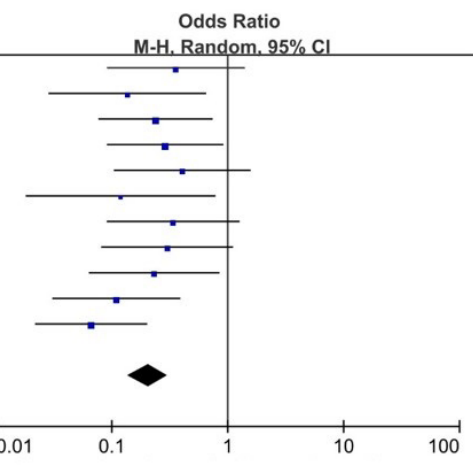
$0.29[0.09,0.92]$

Figure 2 Summary of pooled results regarding death and response. (A) meta-analysis regarding in-hospital death; (B) meta-analysis regarding complete response; (C) meta-analysis regarding partial response; (D) meta-analysis regarding no response. 
Table 2 Summary of pooled results regarding biochemical and clinical variables

\begin{tabular}{|c|c|c|c|c|c|c|c|}
\hline Variable & $\begin{array}{c}\text { Studies } \\
\text { included (n) }\end{array}$ & $\begin{array}{c}\text { Patients } \\
\text { included (n) }\end{array}$ & SMD & $95 \% \mathrm{Cl}$ & Significance, $P$ & \multicolumn{2}{|c|}{ Heterogeneity } \\
\hline \multicolumn{8}{|l|}{ Serum creatinine } \\
\hline After treatment, TCM vs. control & 10 & 462 & -1.78 & $-2.78,-0.78$ & 0.0005 & $<0.00001$ & $95 \%$ \\
\hline TCM, before vs. after & 10 & 474 & -2.56 & $-3.60,-1.52$ & $<0.00001$ & $<0.00001$ & $95 \%$ \\
\hline \multicolumn{8}{|l|}{ Blood urea nitrogen } \\
\hline After treatment, TCM vs. control & 9 & 407 & -1.79 & $-2.87,-0.70$ & 0.001 & $<0.00001$ & $95 \%$ \\
\hline TCM, before vs. after & 9 & 420 & -2.26 & $-3.24,-1.27$ & $<0.00001$ & $<0.00001$ & $93 \%$ \\
\hline Control, before vs. after & 9 & 400 & -0.77 & $-1.53,0.00$ & 0.005 & $<0.00001$ & $92 \%$ \\
\hline TCM, before vs. after & 6 & 266 & -3.66 & $-5.89,-1.42$ & 0.001 & $<0.00001$ & $97 \%$ \\
\hline Control, before vs. after & 6 & 254 & -1.4 & $-2.99,0.18$ & 0.08 & $<0.00001$ & $96 \%$ \\
\hline \multicolumn{8}{|l|}{ Urine volume } \\
\hline After treatment, TCM vs. control & 9 & 400 & 2.95 & $1.07,4.83$ & 0.002 & $<0.00001$ & $97 \%$ \\
\hline TCM, before vs. after & 9 & 410 & 4.72 & $3.12,6.33$ & $<0.00001$ & $<0.00001$ & $95 \%$ \\
\hline Control, before vs. after & 9 & 396 & 3.51 & $1.81,5.22$ & $<0.0001$ & $<0.00001$ & $97 \%$ \\
\hline \multicolumn{8}{|l|}{ Plasma ammonia } \\
\hline After treatment, TCM vs. control & 2 & 64 & -4.83 & $-7.72,-1.95$ & 0.001 & 0.005 & $87 \%$ \\
\hline Control, before vs. after & 3 & 180 & -1.04 & $-1.35,-0.73$ & $<0.00001$ & 0.83 & $0 \%$ \\
\hline
\end{tabular}

SMD, standardized mean difference; $\mathrm{Cl}$, confidence interval; TCM, traditional Chinese medicine.

analyses regarding response.

TCM led to a significantly higher complete response (OR: 3.20, 95\% CI: 2.06-4.97, $\mathrm{P}<0.00001)$. There was no significant heterogeneity $\left(\mathrm{P}=0.35, \mathrm{I}^{2}=10 \%\right)$ (Figure $2 B$ ).

The rate of partial response was not significantly different between TCM and control groups (OR: 1.39, 95\% CI: $0.90-2.15, \mathrm{P}=0.14)$. There was a mild heterogeneity $\left(\mathrm{P}=0.06, \mathrm{I}^{2}=44 \%\right)$ (Figure $\left.2 \mathrm{C}\right)$.

TCM led to a significantly lower no response (OR: 0.20, 95\% CI: $0.14-0.30, \mathrm{P}<0.00001)$. There was no significant heterogeneity $\left(\mathrm{P}=0.59, \mathrm{I}^{2}=0 \%\right)$ (Figure $\left.2 \mathrm{D}\right)$.

\section{Biomedical and clinical variables}

The results of meta-analyses regarding biomedical and clinical variables were summarized in Table 2 .

Serum creatinine, blood urea nitrogen, bilirubin, urine volume, plasma ammonia, and abdominal circumference were significantly improved in TCM group. Serum creatinine, blood urea nitrogen, urine volume, and abdominal circumference were also significantly improved in control group. TCM led to a significantly better improvement in terms of serum creatinine, blood urea nitrogen, bilirubin, urine volume, plasma ammonia, and 
abdominal circumference. In most meta-analyses regarding biomedical and clinical variables, there was significant heterogeneity.

\section{Subgroup analyses}

The results of subgroup analyses were summarized in Table $S 2$.

The subgroup analyses of HRS-1 patients were unavailable due to the absence of relevant data.

The subgroup analyses of HRS-2 patients demonstrated that TCM led to a significantly better improvement in terms of serum creatinine, blood urea nitrogen, and urine volume, and there was statistical significance. There was no significant heterogeneity.

The subgroup analyses of HRS patients diagnosed based on ICA criteria demonstrated that TCM led to a significantly better improvement in terms of serum creatinine, blood urea nitrogen, bilirubin, and urine volume. Heterogeneity remained significant.

\section{Sensitivity analyses}

The results of sensitivity analyses were summarized in Table S3. Heterogeneity remained significant.

\section{Meta-regression}

The results of meta-regression analyses were summarized in Table S4.

Heterogeneity in the meta-analysis regarding serum creatinine in the control group was related to the publication year $(\mathrm{P}=0.022)$. Heterogeneity in the metaanalysis regarding serum creatinine in the TCM group was not related to the publication year, type of HRS, or diagnostic criteria for HRS. Heterogeneity in the metaanalyses regarding blood urea nitrogen and urine volume was not related to the publication year, type of HRS, or diagnostic criteria for HRS.

\section{Discussion}

The present analysis shows that TCM treatment significantly improved the survival and response of patients with HRS compared with conventional treatment. In addition, serum creatinine, blood urea nitrogen, urine volume, and abdominal circumference were improved irrespective of TCM. Finally, the improvement of serum creatinine, blood urea nitrogen, bilirubin, urine volume, serum ammonia, and abdominal circumference was significantly better in TCM group.

China has a long history of TCM application. The theory of TCM is primarily based on the ancient Chinese philosophy. TCM can cure diseases by correcting the maladjustments and restoring self-regulation ability (25). Among the included studies, 7 applied rhubarb $(12,13,15,16,18,22,24), 7$ applied Salvia Miltiorrhiza Bunge $(11,14,18,20,22-24)$, and 2 applied Ligusticum Wallichii $(12,18)$ in TCM group.

Rhubarb has defecation-accelerating, heat-clearing, blood-cooling, toxin-relieving, blood stasis-dredging, dampness-dredging, jaundice-resolving effects according to the Chinese Pharmacopoeia. Rhubarb in the colon can regulate intestinal flora and reduce intestine-derived uremic toxins produced by gut bacteria (26). Rhubarb has a cathartic effect on accelerating the excretion of intestinal toxins, reducing the absorption of toxins, and preventing from liver and kidney damage (27).

Salvia Miltiorrhiza Bunge has a role in blood circulationpromoting, blood stasis-dredging, blood-nourishing, and mind-tranquilizing according to the Chinese Pharmacopoeia. Salvia Miltiorrhiza Bunge has a protective effect on the liver and kidney. Animal study showed that the anti-inflammatory properties of Salvia Miltiorrhiza Bunge extracts might prevent hepatocyte injury possibly by the inhibition of $\mathrm{p} 38$ and nuclear factor $\mathrm{kB}$ signaling in Kupffer cells (28). Salvia Miltiorrhiza Bunge extracts can significantly improve blood urea nitrogen levels associated with impaired renal function and improve renal structural changes (29).

Ligusticum Wallichii has a role in blood circulationpromoting, blood stasis-dredging, "qi" stagnationregulating, pain-alleviating, and dampness-dredging. The mechanism of blood-activating and stasis-resolving medicine on renal hemodynamics is mainly manifested on the levels of vasomotor factors and the action of reninangiotensin, prostaglandins, endothelin, and nitric oxide (30).

Tetramethylpyrazine is an alkaloid found in the roots of Ligusticum Wallichii, which includes the function of antiinflammation, anti-oxidation, anti-atherosclerosis, and anti-fibrosis. Tetramethylpyrazine plays a protective role in hepatic and renal injury caused by ischemia-reperfusion by inhibiting the adhesion and activation of neutrophils mediated by P-selection and the interaction of neutrophils and endothelium (31).

Except for intravenous injection, oral, and external application, enema is also a major route of TCM among the included studies, which can improve the intestinal environment and reduce the production and absorption of 
enterotoxin (10).

There were some limitations in our study. First, although the studies included in the meta-analysis were reported as RCTs, the study quality was poor. The same situation was reported by Teschke et al. (32). Second, the sample size of each included study was relatively small. Third, the diagnostic criteria of HRS were inconsistent among the included studies. Fourth, the type of HRS was unspecified in some studies. Fifth, all the publication regions were in China. Sixth, the heterogeneity was mostly significant in the meta-analyses regarding biochemical and clinical variables. Despite subgroup analysis, sensitivity analysis, and meta-regression analysis were performed, the source of heterogeneity was not well explained. Seventh, only two included studies provided the follow-up time. One study reported that the follow-up time was 2 months. Another study reported that the follow-up time was 3 months. Therefore, it was impossible to explore the effects of followup times on the outcomes.

In conclusions, TCM may be effective for the treatment of HRS. However, our conclusions are hardly generalizable until more well-designed RCTs are performed.

\section{Acknowledgements}

None.

\section{Footnote}

Conflicts of Interest: The authors have no conflicts of interest to declare.

\section{References}

1. Nassar Junior AP, Farias AQ, D' Albuquerque LA, et al. Terlipressin versus norepinephrine in the treatment of hepatorenal syndrome: a systematic review and metaanalysis. PLoS One 2014;9:e107466.

2. Salerno F, Monti V. Hepatorenal syndrome type 1 and bacterial infection: a catastrophic association in patients with cirrhosis. Hepatology 2014;59:1239-41.

3. Egerod Israelsen M, Gluud LL, Krag A. Acute kidney injury and hepatorenal syndrome in cirrhosis. J Gastroenterol Hepatol 2015;30:236-43.

4. Ginès $P$, Schrier RW. Renal failure in cirrhosis. N Engl J Med 2009;361:1279-90.

5. Facciorusso A, Chandar AK, Murad MH, et al. Comparative efficacy of pharmacological strategies for management of type 1 hepatorenal syndrome: a systematic review and network meta-analysis. Lancet Gastroenterol Hepatol 2017;2:94-102.

6. Mattos ÂZ, Mattos AA, Ribeiro RA. Terlipressin versus noradrenaline in the treatment of hepatorenal syndrome: systematic review with meta-analysis and full economic evaluation. Eur J Gastroenterol Hepatol 2016;28:345-51.

7. Zheng JN, Han YJ, Zou TT, et al. Comparative efficacy of vasoconstrictor therapies for type 1 hepatorenal syndrome: a network meta-analysis. Expert Rev Gastroenterol Hepatol 2017;11:1009-18.

8. Nanda A, Reddy R, Safraz H, et al. Pharmacological Therapies for Hepatorenal Syndrome: A Systematic Review and Meta-Analysis. J Clin Gastroenterol 2018;52:360-7.

9. Ginès $\mathrm{P}$, Guevara $M$. Therapy with vasoconstrictor drugs in cirrhosis: The time has arrived. Hepatology 2007;46:1685-7.

10. Branch of Gastrointestinal Disease CAoCM. Expert consensus on TCM diagnosis and treatment of ascites due to cirrhosis (2017). J Clin Hepatol 2017;33:1621-6.

11. Chen LZ, Wu JY. Intravenous injection of Danshen injection combined with diuretic intraperitoneal injection in the treatment of hepatorenal syndrome. Modern Journal of Integrated Traditional Chinese and Western Medicine 2004;13:2693-4.

12. Zou DG, Li HC, Chen QR. Effect of Phentolamine, Ligustrazine and Rhubar on patients with hepatocirrhosis complicated with hepato-renal syndrome. Applied Journal of General Practice 2004;2:499-500.

13. Wu GE, Liu WY, Chang QT, et al. Colonic dialysis combined with Niaoduqing granule enema in the treatment of hepatorenal syndrome. Chinese Journal of Integrated Traditional and Western Nephrology 2007;8:420-1.

14. Gao H, Qu G. Clinical observation on treating cirrhosis and hepatorenal syndrome with integrated Traditional Chinese and Western Medicine. China Foreign Medical Treatment 2009;28:67-8.

15. Yan CW, Guo X, Wang SF, et al. Efficacy of alprostadil in combination with traditional Chinese medicine retention enema in the treatment of hepatorenal syndrome. Chinese Journal of Integrated Traditional and Western Nephrology 2009; 10:733.

16. Tang RG, SUN JQ. Clinical observation of prostaglandin E1 lipid microsphere combined with Rhubarb in treating hepatorenal syndrome. International Journal of Epidemiology and Infectious Disease 2011;38:303-5. 
17. Xiao Q, Zhang Y, Tan SZ. The treatment by warming the kidney and promoting diuresis with Alprostadil in liver cirrhosis complicated with hepato-renal syndrome in 29 cases. J Clini Hepatol 2012;28:189-191.

18. Xing YM. Clinical observation of retention enema with Tradiyional Chinese Medicine combined with Western medicine on hepatorenal syndrome (HRS). Shanxi Journal of Tradiyional Chinese Medicine 2012;28:19-21.

19. Zhu CQ, Chen EJ. Traditional Chinese medicine acupoint topical external application combined with octreotide intravenous injection in treatment of hepatorenal syndrome 27 cases of clinical research. Jiangsu Journal of Tradiyional Chinese Medicine 2012;44:66-7.

20. Tang G, Yang HT. Clinical observation of invigorating spleen and tonifying stomach decoction treating cirrhosis complicated with hepatorenal syndrome (Chinese article). Modern Traditional Chinese Medicine 2013;33:17-8.

21. Xia LM, Yang LW, Tian GB, et al. Alprostadil combined Radix Astragali injection in Treating hepatorenal syndrome. Chinese Journal of Trauma and Disebility Medicine 2013;21:195-6.

22. Qie LX, Fan R. Clinical observation on 37 Cases of hepatorenal syndrome treated with Traditional Chinese Medicine retention enema. Hebei Journal of Traditional Chinese Medicine 2014;36:1482-3.

23. Luo B, Pu J. Clinical evaluation of integrative Chinese and Western medicine in treating cirrhosis and hepatorenal syndrome. Clinical Research and Practice 2016;1:122-3.

24. Zou YZ. Explore the clinical effect of integrated traditional Chinese and Western medicine treatment of hepatorenal syndrome. Cardiovascular Disease Journal of integrated

doi: $10.21037 / \operatorname{tgh} .2018 .08 .02$

Cite this article as: Song T, Guo X, Shao L, Sun M, Romeiro FG, Han D, Bao W, Qi X. A systematic review and metaanalysis of treatment for hepatorenal syndrome with traditional Chinese medicine. Transl Gastroenterol Hepatol 2018;3:57. traditional Chinese and Western Medicine 2017;5:92-3.

25. Wang R, Han D, Sun MY, et al. Efficacy and safety of integration of traditional and Western medicine for the treatment of spontaneous bacterial peritonitis in liver cirrhosis: a systematic review. AME Med J 2017;2:1-27.

26. Shu ZJ, Cao Y, Halmurat U. Gut flora may offer new therapeutic targets for the traditional Chinese medicine enteric dialysis. Expert Opin Ther Targets 2011;15:1147-52.

27. Zhu W, Wang XM. Progress in study on mechanisms of rhubarb in treating chronic renal failure. Zhongguo Zhong Xi Yi Jie He Za Zhi 2005;25:471-5.

28. Yue S, Hu B, Wang Z, et al. Salvia miltiorrhiza compounds protect the liver from acute injury by regulation of $\mathrm{p} 38$ and NFkappaB signaling in Kupffer cells. Pharm Biol 2014;52:1278-85.

29. Park CH, Shin SH, Lee EK, et al. Magnesium Lithospermate B from Salvia miltiorrhiza Bunge Ameliorates Aging-Induced Renal Inflammation and Senescence via NADPH Oxidase-Mediated Reactive Oxygen Generation. Phytother Res 2017;31:721-728.

30. Chen CL, Q; Gao, Hua. Research Progress on the Pharmacological Actions of Blood-activating and Stasisresolving Medicine. Chinese Pharmaceutical Affairs 2011;25:603-5.

31. Chen JL, Zhou T, Chen WX, et al. Effect of tetramethylpyrazine on P-selectin and hepatic/ renal ischemia and reperfusion injury in rats. World $\mathrm{J}$ Gastroenterol 2003;9:1563-6.

32. Teschke R, Wolff A, Frenzel C, et al. Herbal traditional Chinese medicine and its evidence base in gastrointestinal disorders. World J Gastroenterol 2015;21:4466-90. 


\section{Supplementary}

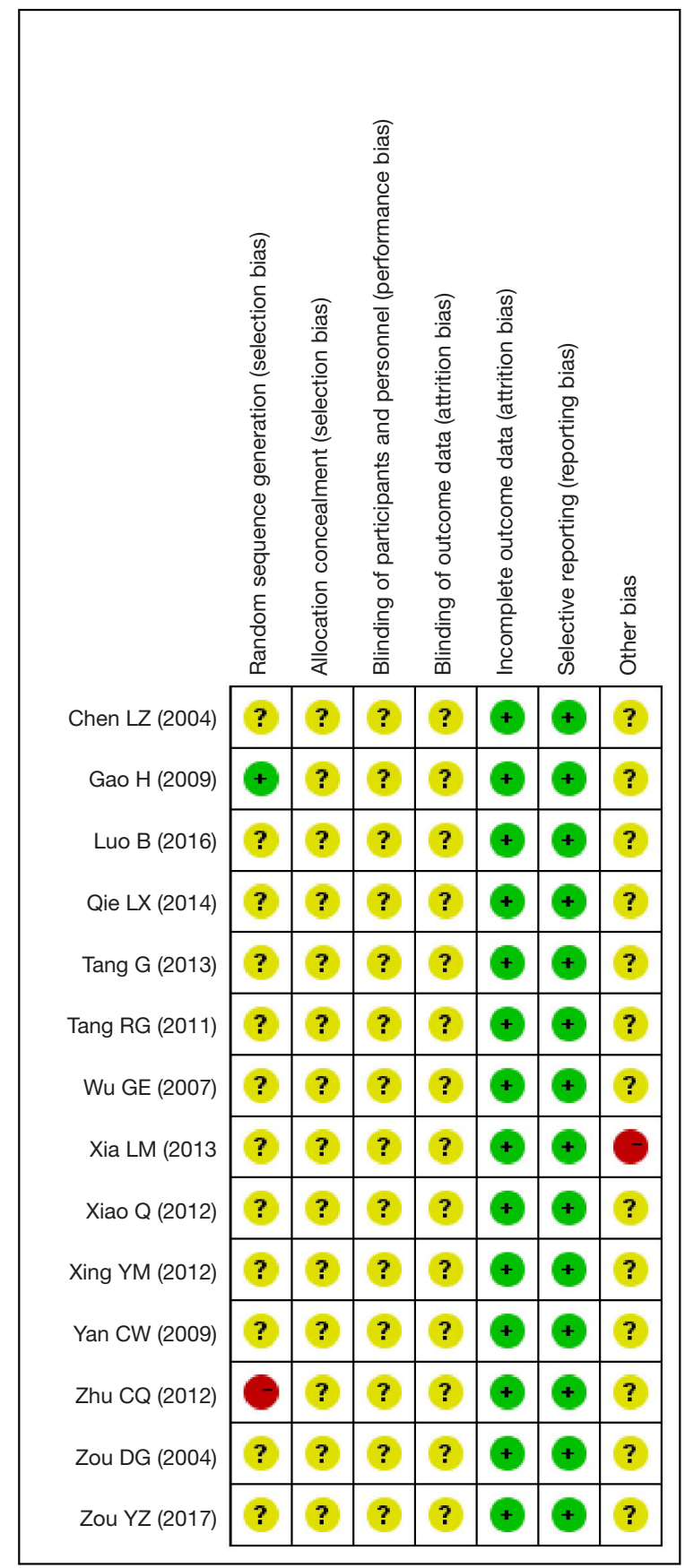

Figure S1 Risk of bias assessment. 
Table S1 Characteristic of the included patients

\begin{tabular}{|c|c|c|c|c|c|c|c|}
\hline Author [year] & Groups & Age & Gender (man/female) & Serum creatinine $(\mu \mathrm{mol} / \mathrm{L})$ & Blood urea nitrogen (mmol/L) & Bilirubin $(\mu \mathrm{mol} / \mathrm{L})$ & Urine volume $(\mathrm{mL} / 24 \mathrm{~h})$ \\
\hline \multirow[t]{3}{*}{ Chen LZ [2004] } & Control [1] & Mean \pm SD: $45 \pm 2.2$ & $18 / 5$ & Mean: 176 & Mean: 29.1 & NA & NA \\
\hline & Control [2] & Mean \pm SD: $46 \pm 1.1$ & $9 / 3$ & Mean: 205 & Mean: 27.3 & NA & NA \\
\hline & TCM & Mean \pm SD: $43 \pm 1.5$ & $16 / 9$ & Mean: 196 & Mean: 26.8 & NA & NA \\
\hline \multirow[t]{2}{*}{ Zou DG [2004] } & Control & Range: $31-71$ & $26 / 4$ & NA & NA & NA & NA \\
\hline & $\mathrm{TCM}$ & Range: 28-69 & $28 / 4$ & $289.09 \pm 15.02$ & $25.23 \pm 4.26$ & NA & NA \\
\hline \multirow[t]{2}{*}{ Wu GE [2007] } & Control & NA & NA & $818.3 \pm 108.5$ & $796.7 \pm 106.1$ & NA & NA \\
\hline & TCM & NA & NA & $825.2 \pm 104.5$ & $38.4 \pm 6.7$ & NA & NA \\
\hline \multirow[t]{2}{*}{ Gao H [2009] } & Control & NA & NA & $174.15 \pm 15.38$ & $15.93 \pm 2.59$ & NA & $484.31 \pm 132.69$ \\
\hline & TCM & NA & NA & $182.07 \pm 37.12$ & $15.51 \pm 3.12$ & NA & $512.43 \pm 144.22$ \\
\hline \multirow[t]{2}{*}{ Yan CW [2009] } & Control & NA & NA & $147.06 \pm 54.31$ & $17.05 \pm 3.21$ & $103.25 \pm 92.23$ & $583.21 \pm 189.25$ \\
\hline & $\mathrm{TCM}$ & NA & NA & $154.08 \pm 47.70$ & $16.30 \pm 4.08$ & $108.83 \pm 90.79$ & $652.21 \pm 135.37$ \\
\hline \multirow[t]{2}{*}{ Tang RG [2011] } & Control & Mean: 51; range: $30-70$ & $9 / 3$ & $463.56 \pm 106.31$ & $21.69 \pm 4.31$ & $59.61 \pm 29.12$ & $583.63 \pm 133.21$ \\
\hline & $\mathrm{TCM}$ & Mean: 48.5; range: 28-69 & $10 / 4$ & $416.23 \pm 53.18$ & $20.93 \pm 4.13$ & $93.84 \pm 30.14$ & $586.34 \pm 130.12$ \\
\hline \multirow[t]{2}{*}{ Xiao Q [2012] } & Control & Mean: 44.7 & $18 / 10$ & $235.7 \pm 63.2$ & $20.9 \pm 5.3$ & $96.3 \pm 4.7$ & $492.5 \pm 142.6$ \\
\hline & $\mathrm{TCM}$ & Mean: 42.1 & $20 / 9$ & $236.8 \pm 67.5$ & $21.4 \pm 5.8$ & $97.6 \pm 5.6$ & $488.6 \pm 135.7$ \\
\hline \multirow[t]{2}{*}{ Xing YM [2012] } & Control & Mean \pm SD: $46 \pm 1.1$ & $16 / 12$ & NA & NA & NA & $440.31 \pm 40.51$ \\
\hline & $\mathrm{TCM}$ & Mean \pm SD: $43 \pm 1.5$ & $18 / 10$ & NA & NA & NA & $450.50 \pm 30.57$ \\
\hline \multirow[t]{2}{*}{ Zhu CQ [2012] } & Control & NA & NA & $189.40 \pm 72.25$ & NA & NA & $484 \pm 162$ \\
\hline & $\mathrm{TCM}$ & NA & NA & $198.39 \pm 57.43$ & NA & NA & $451 \pm 170$ \\
\hline \multirow[t]{2}{*}{ Tang G [2013] } & Control & Mean: 53.7 & $20 / 5$ & $226 \pm 43$ & $21.6 \pm 5.2$ & $71.1 \pm 7.9$ & $483 \pm 19$ \\
\hline & $\mathrm{TCM}$ & Mean: 54.2 & $18 / 7$ & $218 \pm 54.2$ & $22.7 \pm 4.9$ & $75.9 \pm 8.7$ & $471 \pm 18$ \\
\hline \multirow[t]{2}{*}{ Xia LM [2013] } & Control & NA & NA & $146 \pm 53.98$ & $16.15 \pm 3.32$ & NA & $579 \pm 188.95$ \\
\hline & TCM & NA & NA & $153.9 \pm 47.9$ & $16.29 \pm 4.18$ & NA & $652 \pm 135.41$ \\
\hline \multirow[t]{2}{*}{ Qie LX [2014] } & Control & Mean \pm SD: $54.4 \pm 3.8$ & $22 / 15$ & $154.01 \pm 50.45$ & $17.08 \pm 3.24$ & NA & $451.11 \pm 32.45$ \\
\hline & TCM & Mean \pm SD: $52.8 \pm 3.2$ & $20 / 17$ & $147.11 \pm 54.23$ & $17.10 \pm 3.33$ & NA & $440.51 \pm 40.48$ \\
\hline \multirow[t]{2}{*}{ Luo B [2016] } & Control & Mean \pm SD: $56.22 \pm 6.02$ & $21 / 19$ & $111.89 \pm 4.54$ & $11.03 \pm 0.82$ & $145.72 \pm 23.98$ & NA \\
\hline & $\mathrm{TCM}$ & Mean \pm SD: $55.92 \pm 5.83$ & $24 / 26$ & $113.22 \pm 4.82$ & $10.32 \pm 0.73$ & $142.39 \pm 22.47$ & NA \\
\hline \multirow[t]{2}{*}{ Zou YZ [2017] } & Control & Mean:36.5; range:27-71 & $44 / 24$ & NA & NA & NA & NA \\
\hline & $\mathrm{TCM}$ & Mean:38.5; range:23-74 & $40 / 32$ & NA & NA & NA & NA \\
\hline
\end{tabular}

TCM, traditional Chinese medicine; SD, standard deviation; NA, not available. 
Table S2 Subgroup analyses regarding biomedical variables

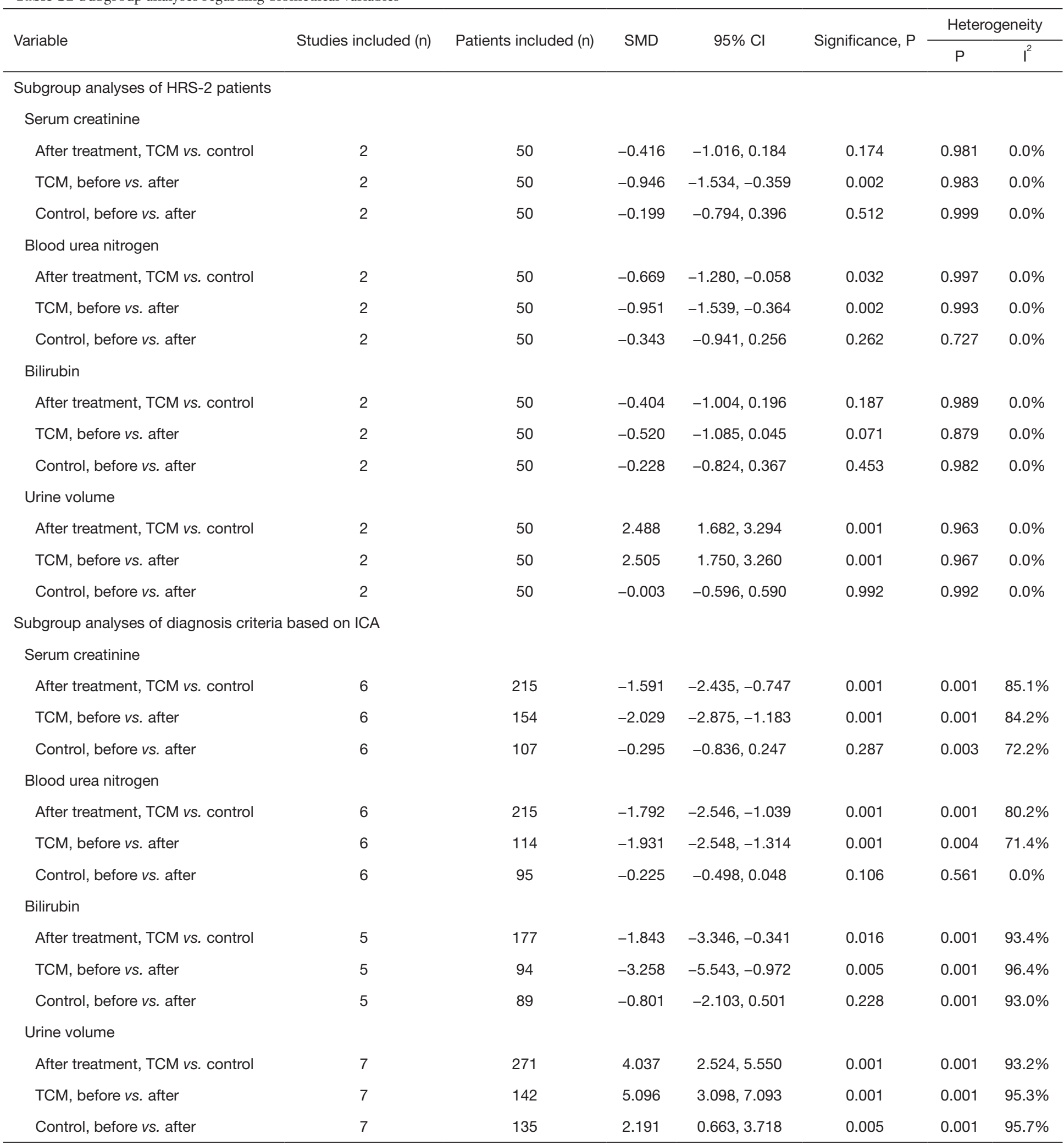

SMD, standardized mean difference; $\mathrm{Cl}$, confidence interval; HRS, hepatorenal syndrome; TCM, traditional Chinese medicine; ICA, International Club of Ascites. 
Table S3 Sensitivity analyses regarding biomedical variables

\begin{tabular}{|c|c|c|c|c|c|c|c|}
\hline \multirow{2}{*}{ Variable } & \multirow{2}{*}{$\begin{array}{c}\text { Studies } \\
\text { included (n) }\end{array}$} & \multirow{2}{*}{$\begin{array}{c}\text { Patients } \\
\text { included (n) }\end{array}$} & \multirow{2}{*}{ SMD } & \multirow{2}{*}{$95 \% \mathrm{Cl}$} & \multirow{2}{*}{$\begin{array}{c}\text { Significance, } \\
\text { P }\end{array}$} & \multicolumn{2}{|c|}{ Heterogeneity } \\
\hline & & & & & & $P$ & $1^{2}$ \\
\hline \multicolumn{8}{|l|}{ Serum creatinine } \\
\hline After treatment, TCM vs. control & 9 & 382 & -1.30 & $-2.07,-0.53$ & 0.0009 & $<0.00001$ & $91 \%$ \\
\hline TCM, before vs. after & 9 & 394 & -1.78 & $-2.48,-1.07$ & $<0.00001$ & $<0.00001$ & $88 \%$ \\
\hline \multicolumn{8}{|l|}{ Blood urea nitrogen } \\
\hline After treatment, TCM vs. control & 8 & 333 & -2.11 & $-2.91,-1.30$ & $<0.00001$ & $<0.00001$ & $87 \%$ \\
\hline TCM, before vs. after & 8 & 340 & -1.74 & $-2.41,-1.06$ & $<0.00001$ & $<0.00001$ & $85 \%$ \\
\hline Control, before vs. after & 8 & 320 & -0.37 & $-0.73,-0.02$ & 0.04 & 0.02 & $57 \%$ \\
\hline TCM, before vs. after & 5 & 208 & -2.51 & $-4.49,-0.53$ & 0.01 & $<0.00001$ & $96 \%$ \\
\hline Control, before vs. after & 5 & 174 & -0.79 & $-2.07,0.49$ & 0.23 & $<0.00001$ & $93 \%$ \\
\hline \multicolumn{8}{|l|}{ Urine volume } \\
\hline After treatment, TCM vs. control & 8 & 350 & 1.54 & $-0.15,3.24$ & 0.07 & $<0.00001$ & $97 \%$ \\
\hline TCM, before vs. after & 8 & 360 & 3.64 & $2.47,4.81$ & $<0.00001$ & $<0.00001$ & $91 \%$ \\
\hline Control, before vs. after & 8 & 346 & 2.21 & $0.71,3.71$ & 0.004 & $<0.00001$ & $96 \%$ \\
\hline
\end{tabular}

SMD, standardized mean difference; $\mathrm{Cl}$, confidence interval; TCM, traditional Chinese medicine.

Table S4 Summary results regarding meta-regression

\begin{tabular}{|c|c|c|c|}
\hline Variable & \multicolumn{3}{|c|}{$\mathrm{P}$} \\
\hline \multicolumn{4}{|l|}{ Serum creatinine } \\
\hline After treatment, TCM vs. control & 0.622 & 0.277 & 0.747 \\
\hline TCM, before vs. after & 0.400 & 0.358 & 0.575 \\
\hline \multicolumn{4}{|l|}{ Blood urea nitrogen } \\
\hline After treatment, TCM vs. control & 0.990 & 0.260 & 0.983 \\
\hline TCM, before vs. after & 0.352 & 0.249 & 0.438 \\
\hline Control, before vs. after & 0.051 & 0.655 & 0.067 \\
\hline \multicolumn{4}{|l|}{ Urine volume } \\
\hline TCM, before vs. after & 0.363 & 0.542 & 0.715 \\
\hline Control, before vs. after & 0.127 & 0.394 & 0.707 \\
\hline
\end{tabular}

HRS, hepatorenal syndrome; TCM, traditional Chinese medicine. 